\title{
POLARIZATION OF KOSZUL CYCLES WITH APPLICATIONS TO POWERS OF EDGE IDEALS OF WHISKER GRAPHS
}

\author{
JÜRGEN HERZOG, TAKAYUKI HIBI, AND AYESHA ASLOOB QURESHI
}

(Communicated by Irena Peeva)

\begin{abstract}
In this paper, we introduce the polarization of Koszul cycles and
\end{abstract} use it to study the depth function of powers of edge ideals of whisker graphs.

\section{INTRODUCTION}

Polarization is a technique to deform an arbitrary monomial ideal $I$ in a polynomial ring $S$ into a squarefree monomial ideal $I^{\wp}$ in a larger polynomial ring $S^{\wp}$, such that $S / I$ is a quotient of $S^{\wp} / I^{\wp}$ modulo a regular sequence of linear forms. The polarized ideal $I^{\wp}$ has a nice property that it has the same graded Betti numbers as I, 9]. Therefore, many questions regarding monomial ideals can be reduced to the study of squarefree monomial ideals. The fact that $I$ and $I^{\wp}$ have the same graded Betti numbers implies that the corresponding Koszul homology modules of the ideal and its polarization have the same vector-space dimension. Therefore, it is natural to ask whether cycles whose homology classes form a basis of the Koszul homology of $I$ can be naturally lifted to cycles representing a basis for the Koszul homology of $I^{\wp}$. In Theorem 1.1 it is shown that this is indeed the case.

In his book [14, Proposition 6.3.2], Villarreal uses polarization to give a simple proof of the fact that the edge ideal of a whisker graph is Cohen-Macaulay. Given a finite simple graph $G$ on the vertex set $V(G)=\left\{x_{1}, \ldots, x_{n}\right\}$ and the edge set $E(G)$, one defines the whisker graph $G^{*}$ of $G$ to be the graph with vertex set $\left\{x_{1}, \ldots, x_{n}, y_{1}, \ldots, y_{n}\right\}$ and edge set $E(G) \cup\left\{\left\{x_{i}, y_{i}\right\}: i=1, \ldots, n\right\}$. By using the results of Section 1, one easily sees that the homology classes of the cycles

$$
x_{i_{1}} \ldots x_{i_{k}} e_{j_{1}} \wedge e_{j_{n-k}} \wedge f_{i_{1}} \wedge \ldots \wedge f_{i_{k}}
$$

with $\mathcal{S}=\left\{i_{1}, \ldots, i_{k}\right\}$ a maximal independent set of $G$ and $\left\{j_{1}, \ldots, j_{n-k}\right\}=$ $V(G) \backslash \mathcal{S}$, form a basis of the Koszul homology $H_{n}\left(x_{1}, \ldots, x_{n}, y_{1}, \ldots, y_{n} ; S^{*} / I\left(G^{*}\right)\right)$. Here $e_{1}, \ldots, e_{n}, f_{1}, \ldots, f_{n}$ is a $S^{*}$-basis of the free module $K_{1}\left(x_{1}, \ldots, x_{n}, y_{1}, \ldots, y_{n}\right.$; $\left.S^{*} / I\left(G^{*}\right)\right)$ with $\partial\left(e_{i}\right)=x_{i}$ and $\partial\left(f_{j}\right)=y_{j}$. A basis cycle, as described in (11), is used in Section 2 in the study of the powers of edge ideals of whisker graphs.

The homological and algebraic behavior of powers of an ideal has been the subject of many research papers in recent years. In particular, the nature of the depth function $f(k)=\operatorname{depth}\left(S / I^{k}\right)$ of a graded ideal $I$ in a polynomial ring $S$ is still quite mysterious. While it is known by a classical result of Brodmann 2 that $f(k)$ for $k \gg 0$ is constant, the behavior of $f(k)$ is not so well understood for initial

Received by the editors November 4, 2013 and, in revised form, January 16, 2014.

2010 Mathematics Subject Classification. Primary 13C13, 13A30, 13F99, 05E40.

Key words and phrases. Polarization, Koszul complex, whisker graphs, edge ideals . 
values of $k$. In [10, it is shown that any non-decreasing bounded integer function $f(k)$ is the depth function of a suitable monomial ideal and it is conjectured that $f(k)$ can be any convergent non-negative integer valued function. In support of this conjecture, it was shown in [1] that $f(k)$ may have arbitrarily many local maxima. On the other hand, it seems that the depth function for the edge ideals behave more tamely. In particular, it is expected that the depth function of an edge ideal is a non-increasing function. This is indicated by the fact that edge ideals satisfy the persistence property for the associated prime ideals of their powers, as shown in [4. Interesting lower bounds for the depth function of an edge ideal have been achieved by Morey [12. On the other hand, even for simple graphs like a line graph or a cycle, the precise depth function is unknown.

In this paper we give an upper bound for the depth function for any connected whisker graph. In fact we show in Theorem 2.2 that for any connected graph $G$ on the vertex set $[n]$, we have $\operatorname{depth}\left(S^{*} / I\left(G^{*}\right)^{k}\right) \leq n-k+1$ for $k=1, \ldots, n$. It can be shown by examples that this upper bound is no longer valid if we drop the assumption that $G$ is connected. For a connected graph this upper bound is obtained by constructing suitable non-vanishing homology classes for the Koszul homology of the powers of $I\left(G^{*}\right)$. The cycles representing these non-vanishing homology classes are obtained as products of certain 1-cycles and an $(n-1)$-cycle which is defined via an independent set of $G$. For showing that the homology of this product of cycles in the corresponding homology group is non-vanishing, we use a combinational fact proved in Proposition 2.1 which says that any connected graph admits a friendly independent set in the sense as described in this proposition. By using results from [5] and [6], we show in Corollary 2.4 that $\operatorname{depth}\left(S^{*} / I\left(G^{*}\right)^{k}\right)=1$ for $k \geq n$ if $G$ is bipartite and $\operatorname{depth}\left(S^{*} / I\left(G^{*}\right)^{k}\right)=0$ for $k \geq n$ if $G$ is non-bipartite.

The upper bound for the depth of the powers of a whisker graph given by our Theorem 2.2 is not always attained. The simplest examples for such a case are the whisker graphs of a 3-cycle or 4-cycle. On the other hand, Villarreal 14, Proposition 6.3.7], has shown that $\operatorname{depth}\left(S^{*} / I\left(G^{*}\right)^{2}\right) \geq n-1$ if $G$ is tree (or even a forest) on the vertex set $[n]$. In Theorem 2.5, we extend the result of Villarreal and show that for any forest $G$ one has $\operatorname{depth}\left(S^{*} / I\left(G^{*}\right)^{k}\right) \geq n-k+1$ for $k=1, \ldots, n$. Together with Theorem 2.2 we conclude that for any tree $G$ we have $\operatorname{depth}\left(S^{*} / I\left(G^{*}\right)^{k}\right)=n-k+1$ for $k=1, \ldots, n$.

\section{Polarization of Koszul cycles}

Let $K$ be a field and $I \subset S=K\left[x_{1}, \ldots, x_{n}\right]$ a monomial ideal in the polynomial ring $S$. We denote as usual by $G(I)$ the unique minimal set of monomial generators of $I$. If $u=x_{1}^{a_{1}} \cdots x_{n}^{a_{n}}$ is a monomial, we call $\mathbf{a}=\left(a_{1}, \ldots, a_{n}\right)$ the multi-degree of $u$ and set $\operatorname{deg}_{x_{i}} u=a_{i}$ for all $i$.

Let $c_{i}=\max \left\{\operatorname{deg}_{x_{i}} u: u \in G(I)\right\}$ for $i=1, \ldots, n$, and let $S^{\wp}$ be the polynomial ring over $K$ in the variables $x_{i j}, i=1, \ldots, n, j=1, \ldots, c_{i}$. The polarization of $I$ is the squarefree monomial $I^{\wp} \subset S^{\wp}$ generated by the monomials $u^{\wp}$ with $u \in G(I)$ where for $u=x_{1}^{a_{1}} \cdots x_{n}^{a_{n}}$ one sets

$$
u^{\wp}=\prod_{i=1, \ldots, n} \prod_{j=1, \ldots, a_{i}} x_{i j} .
$$

We extend this polarization operation to elements in the Koszul complex. Let $K(\mathbf{x} ; I)$ be the Koszul complex of the sequence $\mathbf{x}=x_{1}, \ldots, x_{n}$ with values in $I$. 
Recall that $K_{i}(\mathbf{x})=\bigwedge^{i} F$ where $F=\bigoplus_{j}^{n} S e_{j}$ and where $\partial e_{j}=x_{j}$ for $j=1, \ldots, n$, and that $K(\mathbf{x} ; I)=K(\mathbf{x}) \otimes I$. Thus an element of $K_{i}(\mathbf{x} ; I)$ is of the form

$$
\sum_{J} f_{J} e_{J}
$$

where the sum is taken over all ordered sets $J=\left\{j_{1}<j_{2}<\cdots<j_{i}\right\}$ of cardinality $i$, where $f_{J} \in I$ and where $e_{J}=e_{j_{1}} \wedge e_{j_{2}} \wedge \cdots \wedge e_{j_{i}}$.

Next we consider the Koszul complex $K\left(\mathbf{x}^{\wp} ; I^{\wp}\right)$. Here $\mathbf{x}^{\wp}$ is the sequence

$$
x_{11}, x_{12}, \ldots, x_{1 c_{1}}, x_{21}, \ldots, x_{2 c_{2}}, \ldots, x_{n 1}, \ldots, x_{n c_{n}},
$$

and $K_{i}\left(\mathbf{x}^{\wp}\right)=\bigwedge^{i} G$ where $G=\bigoplus_{i=1, \ldots, n} \bigoplus_{j=1, \ldots, c_{i}} S^{\wp} e_{i j}$.

We call an element $u_{J} e_{J}$ a monomial of $K(\mathbf{x} ; I)$ if $u_{J}$ is a monomial. We set

$$
\operatorname{deg}_{x_{i}}\left(u_{J} e_{J}\right)=\operatorname{deg}_{x_{i}} u_{J}+\delta_{j},
$$

where

$$
\delta_{j}= \begin{cases}1, & \text { if } j \in J \\ 0, & \text { otherwise }\end{cases}
$$

and call

$$
\operatorname{deg}\left(u_{J} e_{J}\right)=\left(\operatorname{deg}_{x_{1}}\left(u_{J} e_{J}\right), \ldots, \operatorname{deg}_{x_{n}}\left(u_{J} e_{J}\right)\right)
$$

the multi-degree of $u_{J} e_{J}$.

For any monomial $u_{J} e_{J}$ of multi-degree $\leq \mathbf{c}$ (componentwise) where $\mathbf{c}=$ $\left(c_{1}, \ldots, c_{n}\right)$, we define the polarization of $u_{J} e_{J}$ to be the monomial

$$
\left(u_{J} e_{J}\right)^{\wp}=u_{J}^{\wp} e_{j_{1} a_{j_{1}}+1} \wedge e_{j_{2} a_{j_{2}}+1} \wedge \cdots \wedge e_{j_{i} a_{j_{i}}+1},
$$

in $K\left(\mathbf{x}^{\wp} ; I^{\wp}\right)$, where $J=\left\{j_{1}<j_{2}<\cdots<j_{i}\right\}$, and $a_{i}=\operatorname{deg}_{x_{i}} u_{J}$.

We extend this polarization operator to an arbitrary multi-homogeneous element $f=\sum_{J} \lambda_{J} u_{J} e_{J}, \lambda_{J} \in K$, of multi-degree $\leq \mathbf{c}$, by setting

$$
f^{\wp}=\sum_{J} \lambda_{J}\left(u_{J} e_{J}\right)^{\wp} .
$$

It follows from [3, Theorem 3.1] that any non-vanishing homology class of $H_{i}(\mathbf{x} ; I)$ can be represented by a multi-homogeneous cycle $z=\sum_{J} \lambda_{J} u_{J} e_{J}$ in $K_{i}(\mathbf{x} ; I)$ with $\operatorname{deg} z \leq \mathbf{c}$. Thus the polarization of such cycles is defined.

The following example demonstrates the polarization of cycles: let $I=$ $\left(x_{1}^{2} x_{2}, x_{1} x_{2}^{2}\right)$. Then $z=x_{1} x_{2}^{2} e_{1}-x_{1}^{2} x_{2} e_{2}$ is a cycle in $K_{1}\left(x_{1}, x_{2} ; I\right)$, and $z^{\wp}=$ $x_{11} x_{21} x_{22} e_{12}-x_{11} x_{12} x_{21} e_{22}$.

With the notation introduced, we have

Theorem 1.1. Let $I \subset S=K\left[x_{1}, \ldots, x_{n}\right]$ be a monomial ideal and let $\mathbf{c}=$ $\left(c_{1}, \ldots, c_{n}\right)$ be the integer vector with $c_{i}=\max \left\{\operatorname{deg}_{x_{i}} u: u \in G(I)\right\}$ for $i=1, \ldots, n$. Let $z_{1}, \ldots, z_{r}$ be multi-homogeneous cycles with multi-degree $\leq \mathbf{c}$, whose homology classes form a $K$-basis of $H_{i}(\mathbf{x} ; I)$. Then the homology classes of the cycles $z_{1}^{\wp}, \ldots, z_{r}^{\wp}$ form a $K$-basis of $H_{i}\left(\mathbf{x}^{\wp} ; I^{\wp}\right)$.

The theorem will be a consequence of the following lemma.

Lemma 1.2. Let $M$ be a finitely graded $S$-module, and assume that $x_{1}$ is a nonzero-divisor modulo $M$. Then there is a natural isomorphism

$$
\varphi: H_{i}\left(x_{1}, \ldots, x_{n} ; M\right) \rightarrow H_{i}\left(x_{2}, \ldots, x_{n} ; \bar{M}\right),
$$


where $\bar{M}$ is the $\bar{S}=S / x_{1} S$-module $M / x_{1} M$. This isomorphism is given as follows: let $z \in Z_{i}\left(x_{1}, \ldots, x_{n} ; M\right)$ and write

$$
z=e_{1} \wedge z_{0}+z_{1}
$$

with $z_{1} \in K_{i}\left(x_{2}, \ldots, x_{n} ; M\right)$. Then the homology class $[z] \in H_{i}\left(x_{1}, \ldots, x_{n} ; M\right)$ is mapped to $\left[\bar{z}_{1}\right] \in H_{i}\left(x_{2}, \ldots, x_{n} ; \bar{M}\right)$, where $\bar{z}_{1}$ is obtained from $z_{1}$ by taking the residue classes of the coefficients of $z_{1}$ modulo $x_{1}$.

Proof. Observe that $\overline{z_{1}}$ is indeed a cycle in $K\left(x_{2}, \ldots, x_{n} ; \bar{M}\right)$, because $0=x_{1} z_{0}-$ $e_{1} \wedge \partial z_{0}+\partial z_{1}$. From this equation it follows that $e_{1} \wedge \partial z_{0}=0$ and hence $\partial \overline{z_{1}}=0$. Next we show that $\varphi$ is well defined. Let $z$ be as in the statement and let $w=z+\partial b$ where $b \in K_{i+1}\left(x_{1}, \ldots, x_{n} ; M\right)$. Let $b=e_{1} \wedge b_{0}+b_{1}$ with $b_{1} \in K_{i+1}\left(x_{2}, \ldots, x_{n} ; M\right)$. Then $w=e_{1} \wedge w_{0}+w_{1}$ where $w_{1}=z_{1}+x_{1} b_{0}+\partial b_{1}$. We have to show that $\left[\bar{w}_{1}\right]=\left[\bar{z}_{1}\right]$. But this is obvious, because $\bar{w}_{1}=\bar{z}_{1}+\partial \bar{b}_{1}$, so that $\bar{w}_{1}$ and $\bar{z}_{1}$ differ only by a boundary in $K_{i}\left(x_{2}, \ldots, x_{n} ; \bar{M}\right)$.

Since $H_{i}\left(x_{1}, \ldots, x_{n} ; M\right) \cong \operatorname{Tor}_{i}^{S}(K ; M)$ and $H_{i}\left(x_{2}, \ldots, x_{n} ; \bar{M}\right) \cong \operatorname{Tor}_{i}^{\bar{S}}(K, \bar{M})$, we conclude that $\operatorname{dim}_{K} H_{i}\left(x_{1}, \ldots, x_{n} ; M\right)=\operatorname{dim}_{K} H_{i}\left(x_{2}, \ldots, x_{n} ; \bar{M}\right)$. Indeed, since $x_{1}$ is non-zero on $M$, the graded minimal free resolution of $\bar{M}$ is obtained from the graded minimal free resolution of $M$ by reduction modulo $x_{1}$. This implies that $\operatorname{dim}_{K} \operatorname{Tor}_{i}^{S}(K ; M)=\operatorname{dim}_{K} \operatorname{Tor}_{i}^{\bar{S}}(K, \bar{M})$. Hence, in order to prove that $\varphi$ is an isomorphism, it suffices to show that $\varphi$ is surjective.

Let $[v] \in H_{i}\left(x_{2}, \ldots, x_{n} ; \bar{M}\right)$. There exists $z_{1} \in K_{i}\left(x_{2}, \ldots, x_{n} ; M\right)$ with $\overline{z_{1}}=v$. It follows that $\partial z_{1}=-x_{1} z_{0}$ for some $z_{0} \in K_{i-1}\left(x_{2}, \ldots, x_{n} ; M\right)$. Since $0=\partial^{2} z_{1}=$ $-x_{1} \partial z_{0}$, we see that $\partial z_{0}=0$. Now we set $z=e_{1} \wedge z_{0}+z_{1}$. Then $z$ is a cycle and $\varphi[z]=[v]$.

Proof of Theorem 1.1. Fix an integer $1 \leq i \leq n$. For each $u \in G(I)$ we define

$$
u^{\prime}= \begin{cases}\left(u / x_{i}\right) y, & \text { if } x_{i}^{2} \mid u, \\ u, & \text { otherwise. }\end{cases}
$$

The element $u^{\prime}$ is called the 1-step polarization of $u$ with respect to the variable $x_{i}$, and the ideal $I^{\prime}=\left(\left\{u^{\prime} \mid u \in G(I)\right\}\right)$ is called a 1-step polarization of $I$. Obviously, the (complete) polarization of $I$ can be obtained by a sequence of 1-step polarization.

Let $I^{\prime}$ be the 1-step polarization of $I$ with respect to $x_{i}$. Without loss of generality, we may assume that $i=1$. We consider the Koszul complex $K\left(y, x_{1}, \ldots, x_{n} ; I^{\prime}\right)$ $=(\bigwedge H) \otimes I^{\prime}$ where $H$ is the free $S[y]$-module with basis $f, e_{1}, \ldots, e_{n}$ and where $\partial f=y$ and $\partial e_{j}=x_{j}$ for $j=1, \ldots, n$. Let $z=\sum_{J} \lambda_{J} u_{J} e_{J}$ be a multi-homogeneous cycle of $K_{i}\left(x_{1}, \ldots, x_{n} ; I\right)$ with $\operatorname{deg} z \leq \mathbf{c}$ whose homology class is non-zero.

We set $z^{\prime}=\sum_{J} \lambda_{J}\left(u_{J} e_{J}\right)^{\prime}$, where

$$
\left(u_{J} e_{J}\right)^{\prime}= \begin{cases}u_{J} e_{J}, & \text { if } x_{1} \nmid u_{J}, \\ u_{J}^{\prime} e_{J}, & \text { if } x_{1} \mid u_{J} \text { and } 1 \notin J, \\ u_{J} e_{J}^{\prime}, & \text { if } x_{1} \mid u_{J} \text { and } 1 \in J .\end{cases}
$$

Here $e_{J}^{\prime}$ is obtained from $e_{J}$ by replacing the factor $e_{1}$ in $e_{J}$ by $f$.

As an example, we consider again the cycle $z=x_{1} x_{2}^{2} e_{1}-x_{1}^{2} x_{2} e_{2}$ in $K_{1}\left(x_{1}, x_{2} ; I\right)$ where $I=\left(x_{1}^{2} x_{2}, x_{1} x_{2}^{2}\right)$. Then $z^{\prime}=x_{1} x_{2}^{2} f-x_{1} y x_{2} e_{2}$.

We claim that $z^{\prime}$ is a cycle in $K_{i}\left(y, x_{1}, \ldots, x_{n} ; I^{\prime}\right)$, and that the map

$$
H_{i}\left(y, x_{1}, \ldots, x_{n} ; I^{\prime}\right) \rightarrow H_{i}\left(x_{1}, \ldots, x_{n} ; I\right), \quad\left[z^{\prime}\right] \mapsto[z]
$$


is an isomorphism. From this claim the theorem follows by induction on the number of 1-step polarizations which are required to obtain the polarization $I^{\wp}$ of $I$.

Proof of the claim: we first show that $z^{\prime}$ is a cycle. We first discuss the case when $\operatorname{deg}_{x_{1}} z \leq 1$.

By the definition of $\left(u_{J} e_{J}\right)^{\prime}$, we have $\left(u_{J} e_{J}\right)^{\prime}=u_{J} e_{J}$, for all $J$. It shows that $z=z^{\prime}$ and hence $z^{\prime}$ is a cycle.

Now we discuss the case when $\operatorname{deg}_{x_{1}} z>1$. Let $z=e_{1} \wedge z_{0}+z_{1}$ and $z^{\prime}=$ $f \wedge z_{0}^{\prime}+z_{1}^{\prime}$ with $z_{1} \in K_{i}\left(x_{2}, \ldots, x_{n} ; I\right)$ and $z_{1}^{\prime} \in K_{i}\left(x_{1}, \ldots, x_{n} ; I^{\prime}\right)$. Moreover, $z_{0}=\sum_{1 \in J} \lambda_{J} u_{J} e_{J \backslash\{1\}}$ and $z_{1}=\sum_{1 \notin J} \lambda_{J} u_{J} e_{J}$. From the definition of $z^{\prime}$ we see that $z_{0}^{\prime}=z_{0}$ and $z_{1}^{\prime}=\sum_{1 \notin J} \lambda_{J} u_{J}^{\prime} e_{J}$ where $u_{J}^{\prime}=y u_{J} / x_{1}$. It implies that $z_{1}^{\prime}=\left(y / x_{1}\right) z_{1}$. By applying $\partial$ on $z^{\prime}$, we get $\partial\left(z^{\prime}\right)=y z_{0}+\partial\left(z_{1}^{\prime}\right)=y z_{0}+\left(y / x_{1}\right) \partial\left(z_{1}\right)$. It shows that $x_{1} \partial\left(z^{\prime}\right)=y \partial(z)=0$. Hence $\partial\left(z^{\prime}\right)=0$.

We first observe that $y-x_{1}$ is a non-zero divisor on $S[y] / I^{\prime}$ and that $I^{\prime} /$ $\left(y-x_{1}\right) I^{\prime}=I$. Therefore, by Lemma 1.2, there exists the isomorphism

$$
\varphi: H_{i}\left(y, x_{1}, \ldots, x_{n}\right)=H_{i}\left(y-x_{1}, x_{1}, \ldots, x_{n} ; I^{\prime}\right) \rightarrow H_{i}\left(x_{1}, \ldots, x_{n} ; I\right) .
$$

Thus it remains to be shown that $\varphi\left(\left[z^{\prime}\right]\right)=[z]$. Applying Lemma 1.2, we write $z^{\prime}=\left(f-e_{1}\right) \wedge w_{0}+w_{1}$.

By definition,

$$
\begin{aligned}
z^{\prime} & =\sum_{x_{1} \nmid u_{J}} u_{J} e_{J}+\sum_{x_{1} \mid u_{J}, 1 \notin J} u_{J}^{\prime} e_{J}+\sum_{x_{1} \mid u_{J}, 1 \in J} u_{J} f \wedge e_{J \backslash\{1\}} \\
& =\sum_{x_{1} \nmid u_{J}} u_{J} e_{J}+\sum_{x_{1} \mid u_{J}, 1 \notin J} u_{J}^{\prime} e_{J}+\sum_{x_{1} \mid u_{J}, 1 \notin J} u_{J}\left(f-e_{1}\right) \wedge e_{J \backslash\{1\}}+\sum_{x_{1} \mid u_{J}, 1 \in J} u_{J} e_{J} .
\end{aligned}
$$

Therefore,

$$
w_{1}=\sum_{x_{1} \nmid u_{J}} u_{J} e_{J}+\sum_{x_{1} \mid u_{J}, 1 \notin J} u_{J}^{\prime} e_{J}+\sum_{x_{1} \mid u_{J}, 1 \in J} u_{J} e_{J} .
$$

From this it follows that $\bar{w}_{1}=z$, which by the definition of $\varphi$ implies that $\varphi\left(\left[z^{\prime}\right]\right)=[z]$, as desired.

Corollary 1.3. Let $I \subset S$ be a monomial ideal as in Theorem 1.1. Let $z_{1}, \ldots, z_{r}$ be multi-homogeneous cycles with multi-degree $\leq \mathbf{c}$, whose homology classes form a $K$-basis of $H_{i}(\mathbf{x} ; S / I)$ for $i \geq 1$. Then the homology classes of the cycles $z_{1}^{\wp}, \ldots, z_{r}^{\wp}$ form a $K$-basis of $H_{i}\left(\mathbf{x}^{\wp} ; S^{\wp} / I^{\wp}\right)$.

Proof. We notice that for $i \geq 1$ there is an isomorphism $\varphi: H_{i}\left(\mathbf{x}^{\wp} ; S^{\wp} / I^{\wp}\right) \rightarrow$ $H_{i+1}\left(\mathbf{x}^{\wp} ; I^{\wp}\right)$ with $\varphi([z])=[\partial(w)]$ and $w \in K\left(\mathbf{x}^{\wp} ; S^{\wp}\right)$ such that $z=w+I^{\wp} K\left(\mathbf{x}^{\wp} ; S^{\wp}\right)$. Since $\partial\left(f^{\wp}\right)=(\partial(f))^{\wp}$ for any multi-homogeneous element $f \in K\left(\mathbf{x} ; S^{\wp}\right)$ with $\operatorname{deg} f \leq \mathbf{c}$, the desired conclusion follows.

As an example for the polarization of Koszul cycles, we consider whisker graphs. Let $G$ be a finite simple graph on the vertex set $[n]=\{1, \ldots, n\}$. The whisker graph $G^{*}$ of $G$ is the graph with the vertex set $V\left(G^{*}\right)=\{1, \ldots, n\} \cup\left\{1^{\prime}, \ldots, n^{\prime}\right\}$ and the edge set $E\left(G^{*}\right)=E(G) \cup\left\{\left\{1,1^{\prime}\right\},\left\{2,2^{\prime}\right\}, \ldots,\left\{n, n^{\prime}\right\}\right\}$.

Figure 1 displays the whisker graph of the graph $G$ with edges $\{1,2\},\{2,3\},\{3,4\}$ and $\{4,2\}$.

Let $K$ be a field. The edge ideal $I(G)$ of $G$ is the monomial ideal in $S=$ $K\left[x_{1}, \ldots, x_{n}\right]$ generated by the monomials $x_{i} x_{j}$ with $\{i, j\} \in E(G)$. We consider 


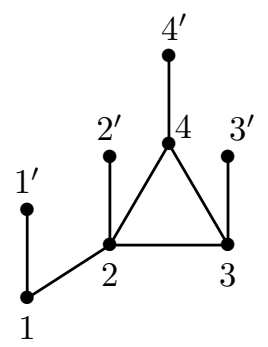

Figure 1

the edge ideal $I\left(G^{*}\right)$ of the whisker graph $G^{*}$ of $G$ as the monomial ideal in $S^{*}=$ $K\left[x_{1}, \ldots, x_{n}, y_{1}, \ldots, y_{n}\right]$ with $I\left(G^{*}\right)=I_{G}+\left(\left\{x_{k} y_{k} \mid k \in[n]\right\}\right)$.

Next, we let $J(G)=\left(I(G), x_{1}^{2}, \ldots, x_{n}^{2}\right)$. Then, obviously, $I\left(G^{*}\right)=J(G)^{\wp}$, where for simplicity we set $x_{i}=x_{i 1}, y_{i}=x_{i 2}$, for $i=1, \ldots, n$. For the polarized Koszul complex of $K\left(x_{1}, \ldots, x_{n} ; I(G)\right)$ we use the notation $e_{i}=e_{i 1}$ and $f_{i}=e_{i 2}$. Given a cycle $\sum_{J} \lambda_{J} u_{J} e_{J} \in K\left(x_{1}, \ldots, x_{n} ; J(G)\right)$ representing a non-zero homology class, the polarized cycle in $K\left(x_{1}, \ldots, x_{n}, y_{1}, \ldots, y_{n} ; I\left(G^{*}\right)\right)$ is given as $\sum_{J} \lambda_{j} u_{j} e_{J^{\prime}}$ where $e_{J^{\prime}}$ is obtained from $e_{J}$ by replacing $e_{j}$ for $j \in J$ by $f_{j}$ if $x_{j} \mid u$.

Note that $H_{n}\left(x_{1}, \ldots, x_{n} ; J(G)\right)$ is minimally generated by the homology classes $\left[u e_{1} \wedge \ldots \wedge e_{n}\right]$ with $u=x_{i_{1}} \ldots x_{i_{k}}$ where $\left\{i_{1}, \ldots, i_{k}\right\}$ is a maximal independent set of $G$. Recall that a subset $\mathcal{S} \subset V(G)$ is an independent set of $G$ if $\{i, j\} \notin E(G)$ for all $i, j \in \mathcal{S}$. The set $\mathcal{S}$ is called a maximal independent set if $\mathcal{S} \cup\{k\}$ is not independent for all $k \notin V(G) \backslash \mathcal{S}$.

It follows from Corollary 1.3. that the elements

$$
x_{i_{1}} \ldots x_{i_{k}} e_{j_{1}} \wedge e_{j_{n-k}} \wedge f_{i_{1}} \wedge \ldots \wedge f_{i_{k}}
$$

form a basis of $H_{n}\left(x_{1}, \ldots, x_{n}, y_{i}, \ldots, y_{n} ; S^{*} / I\left(G^{*}\right)\right)$ where $\mathcal{S}=\left\{i_{1}, \ldots, i_{k}\right\}$ is a maximal independent set of $G$ and $\left\{j_{1}, \ldots, j_{n-k}\right\}=V(G) \backslash \mathcal{S}$.

\section{Powers OF WhISKER GRAPHS}

In this section, we want to study the powers of whisker graphs. For the formulation of the main result we introduce the following concept. Let $G$ be a finite simple graph on $[n]$, and let $\mathcal{S}$ be a maximal independent subset of $V(G)$. We define the graph $\Gamma_{\mathcal{S}}(G)$ with vertex set $V\left(\Gamma_{\mathcal{S}}(G)\right)=\mathcal{S}$ and $\{i, j\} \in E\left(\Gamma_{\mathcal{S}}(G)\right)$ if and only if there exists $k \in V(G) \backslash \mathcal{S}$ such that $\{i, k\},\{j, k\} \in E(G)$.

Proposition 2.1. Let $G$ be a finite simple connected graph. Then there exists an independent set $\mathcal{S}$ such that $\Gamma_{\mathcal{S}}(G)$ is connected.

Proof. Let $\Delta(G)$ be the clique complex of $G$ with cliques $F_{1}, \ldots, F_{r}$. We are going to construct the independent set $\mathcal{S}$ of $G$ as follows. Let $v_{1} \in V\left(F_{1}\right)$. We may assume that $v_{1} \in V\left(F_{i}\right)$ for $i=1, \ldots, t$ and $v_{1} \notin V\left(F_{i}\right)$ for $i>t$. If $t=r$, then we are done. Assume that $t<r$. Since $G$ is connected, there exists $F_{i}$ with $i>t$, say $F_{t+1}$, such that $V\left(F_{t+1}\right) \cap V\left(F_{j}\right) \neq \emptyset$ for some $j \leq t$. Since $F_{t+1}$ is a maximal clique, $V\left(F_{t+1}\right) \not \subset \bigcup_{i=1}^{t} V\left(F_{i}\right)$ because otherwise $v_{1} \in V\left(F_{t+1}\right)$, a contradiction. Hence, we may choose $v_{2} \in V\left(F_{t+1}\right) \backslash \bigcup_{i=1}^{t} V\left(F_{i}\right)$. We may assume that $v_{2} \in V\left(F_{i}\right)$ for $i=t+1, \ldots, s$ and does not belong to any other clique. If $s=r$, then $\Gamma_{\mathcal{S}}(G)$ is a line graph with vertex set $\left\{v_{1}, v_{2}\right\}$. Indeed, $\left\{v_{1}, v_{2}\right\} \notin E(G)$ because the set of neighbors of $v_{1}$ is equal to $\bigcup_{i=1}^{t} F_{i}$ and $v_{2} \notin \bigcup_{i=1}^{t} F_{i}$. On the 
other hand, if $k \in V\left(F_{t+1}\right) \cap V\left(F_{j}\right)$. then $\left\{v_{1}, k\right\},\left\{v_{2}, k\right\} \in E(G)$. Therefore, $\left\{v_{1}, v_{2}\right\} \in E\left(\Gamma_{\mathcal{S}}(G)\right)$.

Consider all $F_{j}$ for $j>s$ such that $V\left(F_{j}\right) \subset \bigcup_{i=1}^{t} V\left(F_{i}\right)$. We may assume that it is the case for $F_{s+1}, \ldots, F_{k}$. If $k=r$, then $\left\{v_{1}, v_{2}\right\}$ is an independent set for $G$, and we are done. If $k<r$, then since $G$ is connected there exists a clique $F_{i}$, say $F_{k+1}$, such that $V\left(F_{k+1}\right) \cap V\left(F_{j}\right) \neq \emptyset$ for some $j<k$ and $V\left(F_{k+1}\right) \not \subset \bigcup_{i=1}^{s} V\left(F_{i}\right)$ $\left(=\bigcup_{i=1}^{k} V\left(F_{i}\right)\right)$. We choose $v_{3} \in V\left(F_{k+1}\right) \backslash \bigcup_{i=1}^{s} V\left(F_{i}\right)$. If $j<t$ then $\left\{v_{1}, v_{3}\right\}$ will be an edge of $\Gamma_{\mathcal{S}}(G)$, and if $t+1 \leq j \leq s$, then $\left\{v_{2}, v_{3}\right\}$ will be an edge of $\Gamma_{\mathcal{S}}(G)$. Proceeding this way, we obtain the desired independent set $\mathcal{S}$ of $G$ such that $\Gamma_{\mathcal{S}}(G)$ is connected.

We call an independent set $\mathcal{S}$ of $G$ friendly if it satisfies the condition that $\Gamma_{\mathcal{S}}(G)$ is connected. For example, if we consider the line graph $L$ on vertex set [4] with edges $\{\{1,2\},\{2,3\},\{3,4\}\}$. Then $\mathcal{S}=\{1,3\}$ is a friendly independent set of $L$ while $\{1,4\}$ is not a friendly independent set of $L$.

Theorem 2.2. Let $G$ be a finite simple connected graph on vertex set $[n]$, and $G^{*}$ be the whisker graph of $G$. Furthermore, let $I\left(G^{*}\right) \subset S^{*}=K\left[x_{1}, \ldots, x_{n}, y_{1}, \ldots, y_{n}\right]$ be the edge ideal of $G^{*}$. Then

$$
\operatorname{depth}\left(S^{*} / I\left(G^{*}\right)^{k}\right) \leq n-k+1, \text { for } k=1, \ldots, n .
$$

Proof. Let $M$ be an $S^{*}$-module and consider the Koszul complex

$$
K(M)=K\left(x_{1}, \ldots, x_{n}, y_{1}, \ldots, y_{n} ; M\right)
$$

with $K_{1}(M)=\bigoplus_{i=1}^{n} M e_{i} \oplus \bigoplus_{j=1}^{n} M f_{j}$ and $\partial e_{i}=x_{i}$ and $\partial f_{j}=y_{j}$, for all $i, j$.

We first show that

$$
H_{2 n-2}\left(I\left(G^{*}\right)^{n}\right) \neq 0
$$

This will imply that $\operatorname{depth}\left(S^{*} / I\left(G^{*}\right)^{n}\right) \leq 1$. To see that the above Koszul homology does not vanish, we proceed as follows.

By Proposition 2.1 we may choose a friendly independent set $\mathcal{S}$ of $G$ with $|\mathcal{S}|=s$. Since $\Gamma_{\mathcal{S}}(G)$ is connected, there exists a spanning tree $T$ of $\Gamma_{\mathcal{S}}(G)$ with $s-1$ edges $\alpha_{1}, \ldots, \alpha_{s-1}$. We may assume that $\alpha_{1}, \ldots, \alpha_{s-1}$ is a leaf order for $T$. In other words, the following conditions are satisfied: (i) $\alpha_{1}$ has a free vertex in $T$, (ii) for each $j>1, \alpha_{j} \cap \alpha_{i} \neq \emptyset$ for some $i<j$ and $\alpha_{j}$ has a free vertex in the tree $T_{j}=\alpha_{1}, \ldots, \alpha_{j}$. Now, we label the vertices of $T$ inductively as follows: 1 is the free vertex of $\alpha_{1}$ in $T_{1}$ and the other vertex in $T_{1}$ is given the label 2. Suppose, the labeling of $T_{j-1}$ is defined. Then we give the new vertex of $T_{j}$, the label $j+1$. Then $\alpha_{1}=\{1,2\}$ and for each $j>1, \alpha_{j}=\left\{i_{j}, j+1\right\}$, where $\left\{i_{j}\right\}=\alpha_{j} \cap \alpha_{i}$.

The following Figure 2 , gives an example of such a labeling.

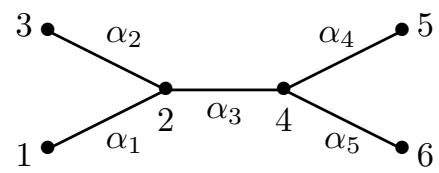

FIGURE 2

According to our labeling of $T$, we have $\mathcal{S}=\{1, \ldots, s\}$. By definition of $\Gamma_{\mathcal{S}}(G)$, there exists for each edge $\alpha_{j}=\left\{i_{j}, j+1\right\} \in E(T)$, a vertex $v_{j} \in\{s+1, \ldots, n\}$ such that $\left\{i_{j}, v_{j}\right\},\left\{v_{j}, j+1\right\} \in E(G)$. Then $z_{j}=x_{i_{j}} x_{v_{j}} e_{j+1}-x_{j+1} x_{v_{j}} e_{i_{j}}$ is a cycle belonging to $Z_{1}\left(I\left(G^{*}\right)\right)$. 
Furthermore, for each $k \in\{s+1, \ldots, n\}$, we choose $j_{k} \in \mathcal{S}$ such that $\left\{k, j_{k}\right\} \in$ $E(G)$. Then $z_{k}=x_{k} x_{j_{k}} f_{k}-x_{k} y_{k} e_{j_{k}}$ is a cycle belonging to $Z_{1}\left(I\left(G^{*}\right)\right)$. This gives $n-s$ such cycles.

Let

$$
c=\prod_{i=1}^{s} x_{i} e_{s+1} \wedge \ldots \wedge e_{n} \wedge f_{1} \wedge \ldots \wedge f_{s}
$$

Note that by (2), $c$ is a cycle in $Z_{n}\left(S^{*} / I\left(G^{*}\right)\right)$ whose homology class $[c]$ in $H_{n}\left(S^{*} / I\left(G^{*}\right)\right)$ is non-zero. In particular, $[\partial(c)]$ is a non-zero homology class in $H_{n-1}\left(I\left(G^{*}\right)\right)$.

Let

$$
a=c \wedge z_{1} \wedge \ldots \wedge z_{s-1} \wedge z_{s+1} \wedge \ldots \wedge z_{n} .
$$

Observe that $a \in K_{2 n-1}\left(I\left(G^{*}\right)^{n-1}\right)$. We set $z=\partial(a)$. Then $z \in Z_{2 n-2}\left(I\left(G^{*}\right)^{n}\right)$. Indeed, $z=\partial(c) \wedge z_{1} \wedge \ldots \wedge z_{s-1} \wedge z_{s+1} \wedge \ldots \wedge z_{n}$, and it has coefficients in $I\left(G^{*}\right)^{n}$ because $\partial(c)$ and each $z_{i}$ has coefficients in $I\left(G^{*}\right)$.

We claim that $[z]$ is a non-zero homology class in $H_{2 n-2}\left(I\left(G^{*}\right)^{n}\right)$. To prove the claim, we show that $z$ is not a boundary, that is, there does not exist any $b \in$ $K_{2 n-1}\left(I\left(G^{*}\right)^{n}\right)$ such that $z=\partial b$. On the contrary, assume that such $b$ exists. Then $\partial(b)=\partial(a)=z$ implies $\partial(a-b)=0$ which gives $a-b \in Z_{2 n-1}\left(I\left(G^{*}\right)^{n-1}\right)$. Then, there exists $b^{\prime} \in K_{2 n}\left(S^{*}\right)$ such that $\partial\left(b^{\prime}\right)=a-b$ where $b^{\prime}=v e_{1} \wedge \ldots \wedge e_{n} \wedge f_{1} \wedge \ldots \wedge f_{n}$ and $v$ is a monomial in $S^{*}$ because all cycles under consideration are $\mathbb{Z}^{2 n}$-graded.

Note that

$$
w=\left(\prod_{i=1}^{s} x_{i}\right)\left(\prod_{k=s+1}^{n} x_{k} x_{j_{k}}\right)\left(\prod_{j=1}^{s-1} x_{i_{j}} x_{v_{j}}\right) e_{2} \wedge \cdots \wedge e_{n} \wedge f_{1} \wedge \cdots \wedge f_{n}
$$

with $i_{j}, j_{k} \in \mathcal{S}, k, v_{j} \in\{s+1, \ldots, n\}$ is a non-zero term of $a$ and it is not cancelled by any other term of $a$ because the product $e_{2} \wedge \cdots \wedge e_{n} \wedge f_{1} \wedge \cdots \wedge f_{n}$ appears only once in the expansion of $a$. To see this, consider

$$
c \wedge z_{s+1} \wedge \cdots \wedge z_{n}=\left(\prod_{i=1}^{s} x_{i}\right)\left(\prod_{k=s+1}^{n} x_{k} x_{j_{k}}\right) e_{s+1} \wedge \cdots \wedge e_{n} \wedge f_{1} \wedge \cdots \wedge f_{n} .
$$

Therefore, it follows that the term $w$ appears only once in the expansion of $a$ if $e_{2} \wedge \cdots \wedge e_{s-1}$ appears only once in the expansion of $z_{1} \wedge \cdots \wedge z_{s-1}$. Now to see this, we write $z_{j}=g_{j}-h_{j}$, where $g_{j}=x_{i_{j}} x_{v_{j}} e_{j+1}$ and $h_{j}=x_{j+1} x_{v_{j}} e_{i_{j}}$ for $j=1, \ldots, s-1$. Note that for $1 \leq t \leq s-1$ the wedge product $z_{1} \wedge \cdots \wedge z_{t}$ is a linear combination of $g_{i_{1}} \wedge \cdots \wedge g_{i_{k}} \wedge h_{j_{1}} \wedge \cdots \wedge h_{j_{t}}$ with $\left\{i_{1}, \ldots, i_{k}\right\} \cup\left\{j_{1}, \ldots, j_{t}\right\}=\{1, \ldots, t\}$. We prove by induction on $t$ that among these terms, $g_{1} \wedge \cdots \wedge g_{t}$ is the only term that does not contain $e_{1}$. For $t=1$, the assertion is trivial. Now let $t>1$ and assume that the only term that does not contain $e_{1}$ is $g_{1} \wedge \cdots \wedge g_{t-1}$. Then, the only terms of $z_{1} \wedge \cdots \wedge z_{t}$ which do not contain $e_{1}$ are either $g_{1} \wedge \cdots \wedge g_{t}$ or $g_{1} \wedge \cdots \wedge g_{t-1} \wedge h_{t}$. However, by the definition of the cycles $z_{j}$ given in terms of the tree $T$, it follows that $i_{t}=\{2, \ldots, t-1\}$. Therefore, $g_{1} \wedge \cdots \wedge g_{t-1} \wedge h_{t}=0$.

Next, we show that $a \in K_{2 n-1}\left(I\left(G^{*}\right)^{n-1}\right) \backslash K_{2 n-1}\left(I\left(G^{*}\right)^{n}\right)$. For this it suffices to show that $w \in K_{2 n-1}\left(I\left(G^{*}\right)^{n-1}\right) \backslash K_{2 n-1}\left(I\left(G^{*}\right)^{n}\right)$, because $w$ is a non-zero term of $a$ which does not cancel against any other term in $a$, as we have just seen. In fact, $\left(\prod_{i=1}^{s} x_{i}\right)\left(\prod_{k=s+1}^{n} x_{k} x_{j_{k}}\right)\left(\prod_{j=1}^{s-1} x_{i_{j}} x_{v_{j}}\right)$ which is a coefficient of $w$, there are $n-1$ terms with indices in $\{s+1, \ldots, n\}$ and $n+s-1$ terms with indices in 
$\mathcal{S}=\{1, \ldots, s\}$. Since $\mathcal{S}$ is a maximal independent set, this implies that $w$ contains a product of exactly $n-1$ generators of $I\left(G^{*}\right)$.

Since all coefficients of $b=a-\partial\left(b^{\prime}\right)$ are in $I\left(G^{*}\right)^{n}$ and the term $w$ which appears in the expansion of $a$ does not have coefficient in $I\left(G^{*}\right)^{n}, w$ must be cancelled by some term of $\partial\left(b^{\prime}\right)$. This gives

$v x_{1} e_{2} \wedge \cdots \wedge e_{n} f_{1} \wedge \ldots \wedge f_{n}=\left(\prod_{i=1}^{s} x_{i}\right)\left(\prod_{k=s+1}^{n} x_{k} x_{j_{k}}\right)\left(\prod_{j=1}^{s-1} x_{i_{j}} x_{v_{j}}\right) e_{2} \wedge \cdots \wedge e_{n} \wedge f_{1} \wedge \cdots \wedge f_{n}$,

which implies

$$
v=\left(\prod_{i=2}^{s} x_{i}\right)\left(\prod_{k=s+1}^{n} x_{k} x_{j_{k}}\right)\left(\prod_{j=1}^{s-1} x_{i_{j}} x_{v_{j}}\right) \in I\left(G^{*}\right)^{n-1} .
$$

The coefficient of the term $v y_{n} e_{1} \wedge \ldots \wedge e_{n} f_{1} \wedge \cdots \wedge f_{n-1}$ which appears in the expansion of $\partial\left(b^{\prime}\right)$ does not belong to $I\left(G^{*}\right)^{n}$ because $x_{n}$ is the only neighbor of $y_{n}$. Also the term $v y_{n} e_{1} \wedge \ldots \wedge e_{n} f_{1} \wedge \cdots \wedge f_{n-1}$ is not cancelled by any of the terms of $a$ because from (3) we can see that all terms of $a$ contain the wedge product $f_{1} \wedge \cdots \wedge f_{n}$ as a factor. Hence, our assumption that $z$ is a boundary leads us to contradiction.

For simplicity of notation, we set $z_{i}^{\prime}=z_{i}$ for $i=1, \ldots, s-1$ and $z_{i}^{\prime}=z_{i+1}$ for $i=s, \ldots, n-1$. Note that $\partial(c) \wedge z_{1}^{\prime} \wedge \cdots \wedge z_{k-1}^{\prime} \in Z_{n+k-2}\left(I\left(G^{*}\right)^{k}\right)$. We claim that this cycle is not a boundary in $K\left(I\left(G^{*}\right)^{k}\right)$. This then implies that $\operatorname{depth}\left(S^{*} / I\left(G^{*}\right)^{k}\right) \leq n+k-1$ since $H_{n+k-1}\left(S^{*} / I\left(G^{*}\right)^{k}\right) \cong H_{n+k-2}\left(I\left(G^{*}\right)^{k}\right) \neq 0$.

In order to prove the claim, assume that there exists $b \in K_{n+k-1}\left(I\left(G^{*}\right)^{k}\right)$ such that $\partial(b)=\partial(c) \wedge z_{1}^{\prime} \wedge \cdots \wedge z_{k-1}^{\prime}$. Let $b^{\prime}=b \wedge z_{k}^{\prime} \wedge \cdots \wedge z_{n-1}^{\prime}$. Then $b^{\prime} \in K_{2 n-1}\left(I\left(G^{*}\right)^{n}\right)$ and $\partial\left(b^{\prime}\right)=\partial(b) \wedge z_{k}^{\prime} \wedge \cdots \wedge z_{n-1}^{\prime}=z$, a contradiction.

Our hypothesis of Theorem 2.2, which requires that $G$ is connected, is needed. For example, if we take the disconnected graph $G$ on vertex [4] with edge $\{\{1,2\},\{3,4\}\}$, then $\operatorname{depth}\left(S^{*} / I\left(G^{*}\right)^{4}\right)=2$.

Remark 2.3. Let $I$ be an arbitrary monomial ideal in $K\left[x_{1}, \ldots, x_{n}, y_{1}, \ldots, y_{n}\right]$. In [11, Theorem 3.3], it is shown that $\operatorname{depth}\left(S / I^{k}\right) \leq 2 n-k+1$ for $k=1, \ldots, r$, where $r<2 n$ is a number depending on $I$. Comparing this result with our Theorem 2.2 , where $I$ is the edge ideal of a whisker graph, our bound is about half of the bound which is valid for general monomial ideals.

Corollary 2.4. Let $G$ be a finite simple connected graph on vertex set $[n], G^{*}$ be the whisker graph of $G$, and $I\left(G^{*}\right) \subset S^{*}$ be the edge ideal of $G^{*}$. If $G$ is bipartite, then $\operatorname{depth}\left(S^{*} / I\left(G^{*}\right)^{k}\right)=1$ for all $k \geq n$, and if $G$ is non-bipartite, then $\operatorname{depth}\left(S^{*} / I\left(G^{*}\right)^{k}\right)=0$ for all $k \geq n$.

Proof. Suppose first that $G$ is bipartite. Then $G^{*}$ is bipartite as well. It follows from [13. Theorem 5.9] that the $\operatorname{depth}\left(S^{*} / I\left(G^{*}\right)^{k}\right) \geq 1$ for all $k$. Thus our Theorem 2.2 implies that $\operatorname{depth}\left(S^{*} / I\left(G^{*}\right)^{n}\right)=1$. On the other hand, since the Rees ring $R\left(I\left(G^{*}\right)\right.$ ) of $I\left(G^{*}\right)$ is Cohen-Macaulay (see for example [7, Corollary 5.20]), the result of Eisenbud and Huneke [6, Proposition 3.3] yields the desired conclusion.

Now let $G$ be a non-bipartite graph. It follows from [5, Corollary 4.3], applied to our case, that $\operatorname{Ass}\left(S^{*} / I\left(G^{*}\right)^{k}\right)=\operatorname{Ass}\left(S^{*} / I\left(G^{*}\right)^{n}\right)$ for all $k \geq n$. On the other hand, since $G$ is non-bipartite, we know by [5, Corollary 3.4] that $\operatorname{depth}\left(S^{*} / I\left(G^{*}\right)^{k}\right)=0$ for $k \gg 0$. This implies that $\operatorname{depth}\left(S^{*} / I\left(G^{*}\right)^{k}\right)=0$ for all $k \geq n$. 
In general, the upper bounds for the depth of the powers of the edge ideal of a whisker graph given in Theorem 2.2 are not attained. For example, if $G$ is a 3 -cycle then $\operatorname{depth}\left(S^{*} / I\left(G^{*}\right)\right)=3$ and $\operatorname{depth}\left(S^{*} / I\left(G^{*}\right)^{k}\right)=0$ for all $k \geq 2$. Even if $G$ is bipartite, this bound is not attained. For example, if $G$ is a 4-cycle, then $\operatorname{depth}\left(S^{*} / I\left(G^{*}\right)\right)=4, \operatorname{depth}\left(S^{*} / I\left(G^{*}\right)^{2}\right)=3$ and $\operatorname{depth}\left(S^{*} / I\left(G^{*}\right)^{k}\right)=1$ for $k \geq 3$.

On the other hand, Villarreal showed [14, Proposition 6.3.7], that if $G$ is a forest, then $\operatorname{depth}\left(S^{*} / I\left(G^{*}\right)^{2}\right) \geq n-1$. Together with our Theorem 2.2 it follows that $\operatorname{depth}\left(S^{*} / I\left(G^{*}\right)^{2}\right)=n-1$. By using the arguments applied in Villarreal's proof, we now show more generally

Theorem 2.5. Let $G$ be a forest on $n$ vertices and let

$$
I=I\left(G^{*}\right) \subset S^{*}=K\left[x_{1}, \ldots, x_{n}, y_{1}, \ldots, y_{n}\right]
$$

be the edge ideal of $G^{*}$. Then

$$
\operatorname{depth}\left(S^{*} / I^{k}\right) \geq n-k+1 \quad \text { for } k=1, \ldots, n .
$$

Proof. We may assume that $G$ contains at least one edge, otherwise $G$ consists of only isolated vertices and in this case $I$ is a complete intersection and the formula is known, see for example [8, Corollary 1.3]. Our assumption implies that $n \geq 2$. We prove the desired inequality by induction on $k+n$. If $k=1$ then $k+n \geq 3$. For $k+n=3$ we have $k=1$ and $n=2$ and $\operatorname{depth}\left(S^{*} / I\right)=2$ since $I$ is a CohenMacaulay ideal of height 2 .

Let $k+n>3$. Then $n \geq 2$. We may assume that $x_{n}$ is a free vertex of the forest $G$ with $\left\{x_{n-1}, x_{n}\right\} \in E(G)$. Following the notation used in the proof [14, Proposition 6.3.7], we denote by $J$ the ideal which is obtained from $I$ by substituting $x_{n}=0$, and by $L$ the ideal which is obtained from $J$ by substituting $x_{n-1}=0$. Furthermore, we set $K=\left(J^{k}, x_{n-1} x_{n}, x_{n} y_{n}\right)$. Since $\left(J^{k}, x_{n-1} x_{n}\right): x_{n}=\left(L^{k}, x_{n-1}\right)$, we obtain the exact sequence

$$
0 \rightarrow S^{*} /\left(L^{k}, x_{n-1}\right) \rightarrow S^{*} /\left(J^{k}, x_{n-1} x_{n}\right) \rightarrow S^{*} /\left(J^{k}, x_{n}\right) \rightarrow 0
$$

Since $J$ is the edge ideal of a whisker forest on $n-1$ vertices and $L$ is the edge ideal of the whisker forest on $n-2$ vertices, our induction hypothesis implies that

$$
\operatorname{depth}\left(S^{*} /\left(L^{k}, x_{n-1}\right)\right) \geq n-k+2 \text { and } \operatorname{depth}\left(S^{*} /\left(J^{k}, x_{n}\right)\right) \geq n-k+1 .
$$

This implies that

$$
\operatorname{depth}\left(S^{*} /\left(J^{k}, x_{n-1} x_{n}\right)\right) \geq n-k+1
$$

Since $\left(J^{k}, x_{n-1} x_{n}\right): x_{n} y_{n}=\left(L^{k}, x_{n-1}\right)$, we obtain the exact sequence

$$
0 \rightarrow S^{*} /\left(L^{k}, x_{n-1}\right) \rightarrow S^{*} /\left(J^{k}, x_{n-1} x_{n}\right) \rightarrow S^{*} / K \rightarrow 0 .
$$

From (4) and (5), we obtain

$$
\operatorname{depth}\left(S^{*} / K\right) \geq n-k+1 .
$$

Note that $\left(I^{k}, x_{n} y_{n}\right)=\left(J, x_{n-1} x_{n}\right)^{k}+\left(x_{n} y_{n}\right)$. Therefore, $\left(I^{k}, x_{n} y_{n}\right): x_{n-1} x_{n}=$ $\left(J, x_{n-1} x_{n}\right)^{k}: x_{n-1} x_{n}+\left(y_{n}\right)$. Since $\left(J, x_{n-1} x_{n}\right)$ is the edge ideal of the graph for which $\{n-1, n\}$ is an edge with free vertex $n$, it follows by a result of Morey [12, Lemma 2.10] that $\left(J, x_{n-1} x_{n}\right)^{k}: x_{n-1} x_{n}=\left(J, x_{n-1} x_{n}\right)^{k-1}$. Therefore, altogether we have that $\left(I^{k}, x_{n} y_{n}\right): x_{n-1} x_{n}=\left(J, x_{n-1} x_{n}\right)^{k-1}+\left(y_{n}\right)$. Thus, we obtain the exact sequence

$$
0 \rightarrow S^{*} /\left(\left(J, x_{n-1 x_{n}}\right)^{k-1}+\left(y_{n}\right)\right) \rightarrow S^{*} /\left(I^{k}, x_{n} y_{n}\right) \rightarrow S^{*} / K \rightarrow 0 .
$$


We claim that for $k=2, \ldots, n$ we have $\operatorname{depth}\left(S^{*} /\left(J, x_{n-1} x_{n}\right)^{k-1}\right) \geq n-k+2$. Therefore, (6) and (7) implies

$$
\operatorname{depth}\left(S^{*} /\left(I^{k}, x_{n} y_{n}\right)\right) \geq n-k+1 .
$$

By using (8) and our induction hypothesis, the exact sequence

$$
0 \rightarrow S^{*} / I^{k-1} \rightarrow S^{*} / I^{k} \rightarrow S^{*} /\left(I^{k}, x_{n} y_{n}\right) \rightarrow 0 .
$$

yields that $\operatorname{depth}\left(S^{*} / I^{k}\right) \geq n-k+1$ and proves our theorem.

It remains to prove the claim. For that we use induction on $k$. For $k=2$, this inequality is shown in the proof of [14, Proposition 6.3.7]. Suppose that $k>2$. Since $\left(J, x_{n-1} x_{n}\right)$ is the edge ideal of a tree with free vertex $n$, we may apply [12, Lemma 2.10], and obtain the exact sequence

$$
0 \rightarrow S^{*} /\left(J, x_{n-1 x_{n}}\right)^{k-2} \rightarrow S^{*} /\left(J, x_{n-1} x_{n}\right)^{k-1} \rightarrow S^{*} /\left(J^{k-1}, x_{n-1} x_{n}\right) \rightarrow 0 .
$$

By our induction hypothesis, $\operatorname{depth}\left(S^{*} /\left(J, x_{n-1 x_{n}}\right)^{k-2}\right) \geq n-k+3$ and (4) applied for $k-1$ yields $\operatorname{depth}\left(S^{*} /\left(J, x_{n-1 x_{n}}\right)^{k-2}\right) \geq n-k+2$. Therefore, it follows that $\operatorname{depth}\left(S^{*} /\left(J, x_{n-1} x_{n}\right)^{k-1}\right) \geq n-k+2$, as desired.

By combining Theorem 2.2 and Theorem 2.5, we obtain

Corollary 2.6. Let $G$ be a tree. Then

$$
\operatorname{depth}\left(S^{*} / I\left(G^{*}\right)^{k}\right)=n-k+1 \quad \text { for } k=1, \ldots, n .
$$

More generally, we expect that if $G$ is a forest with $m$ connected components, then

$$
\operatorname{depth}\left(S^{*} / I\left(G^{*}\right)^{k}\right)= \begin{cases}n-k+1, & \text { if } k \leq n-m+1 \\ m, & \text { if } k \geq n-m+1\end{cases}
$$

\section{REFERENCES}

[1] S. Bandari, J. Herzog, and T. Hibi, Monomial ideals whose depth function has any given number of strict local maxima, Ark. Mat. DOI 10.1007/s11512-013-0184-1

[2] M. Brodmann, The asymptotic nature of the analytic spread, Math. Proc. Cambridge Philos. Soc. 86 (1979), no. 1, 35-39, DOI 10.1017/S030500410000061X. MR530808(81e:13003)

[3] Winfried Bruns and Jürgen Herzog, Cohen-Macaulay rings, Cambridge Studies in Advanced Mathematics, vol. 39, Cambridge University Press, Cambridge, 1993. MR1251956 (95h:13020)

[4] José Martínez-Bernal, Susan Morey, and Rafael H. Villarreal, Associated primes of powers of edge ideals, Collect. Math. 63 (2012), no. 3, 361-374, DOI 10.1007/s13348-011-0045-9. MR2957976

[5] Janet Chen, Susan Morey, and Anne Sung, The stable set of associated primes of the ideal of a graph, Rocky Mountain J. Math. 32 (2002), no. 1, 71-89, DOI 10.1216/rmjm/1030539608. MR:1911348 (2003e:05132)

[6] David Eisenbud and Craig Huneke, Cohen-Macaulay Rees algebras and their specialization, J. Algebra 81 (1983), no. 1, 202-224, DOI 10.1016/0021-8693(83)90216-8. MR696134 (84h:13030)

[7] Viviana Ene and Jürgen Herzog, Gröbner bases in commutative algebra, Graduate Studies in Mathematics, vol. 130, American Mathematical Society, Providence, RI, 2012. MR2850142

[8] Elena Guardo and Adam Van Tuyl, Powers of complete intersections: graded Betti numbers and applications, Illinois J. Math. 49 (2005), no. 1, 265-279 (electronic). MR.2157379 (2006k:13035)

[9] Jürgen Herzog and Takayuki Hibi, Monomial ideals, Graduate Texts in Mathematics, vol. 260, Springer-Verlag London Ltd., London, 2011. MR2724673 (2011k:13019)

[10] Jürgen Herzog and Takayuki Hibi, The depth of powers of an ideal, J. Algebra 291 (2005), no. 2, 534-550, DOI 10.1016/j.jalgebra.2005.04.007. MR2163482 (2006h:13023) 
[11] J. Herzog and A. A. Qureshi, Persistence and stability properties of powers of ideals. (To appear in J. of App. and Pure Algebra.)

[12] Susan Morey, Depths of powers of the edge ideal of a tree, Comm. Algebra 38 (2010), no. 11, 4042-4055, DOI 10.1080/00927870903286900. MR2764849 (2011m:13039)

[13] Aron Simis, Wolmer V. Vasconcelos, and Rafael H. Villarreal, On the ideal theory of graphs, J. Algebra 167 (1994), no. 2, 389-416, DOI 10.1006/jabr.1994.1192. MR1283294(95e:13002)

[14] Rafael H. Villarreal, Monomial algebras, Monographs and Textbooks in Pure and Applied Mathematics, vol. 238, Marcel Dekker Inc., New York, 2001. MR.1800904 (2002c:13001)

Fachbereich Mathematik, Universität Duisburg-Essen, Campus Essen, 45117 Essen, GERManY

E-mail address: juergen.herzog@uni-essen.de

Department of Pure and Applied Mathematics, Graduate School of Information Science and Technology, Osaka University, Toyonaka, Osaka 560-0043, Japan

E-mail address: hibi@math.sci.osaka-u.ac.jp

The Abdus Salam International Center of Theoretical Physics, Trieste, Italy

Current address: Department of Pure and Applied Mathematics, Graduate School of Information Science and Technology, Osaka University, Toyonaka, Osaka 560-0043, Japan

E-mail address: aqureshi@cr.math.sci.osaka-u.ac.jp 